\title{
Rare Complication of Silicone Fluid Injection in Lower Leg Cared by Micronized Acellular Dermal Matrix
}

\author{
Seung Ki Ahn' ${ }^{1}$, Hwan Jun $\mathrm{Choi}^{1}$, Si Hyung Jang ${ }^{2}$ \\ ${ }^{1}$ Department of Plastic and Reconstructive Surgery, College of Medicine, Soonchunhyang University, Cheonan; ${ }^{2}$ Department of Pathology, College of Medicine, \\ Soonchunhyang University, Cheonan, Korea
}

\begin{abstract}
Earlier, in our country, massive contaminated industrial silicone was injected into humans for contour expansion. This caused many complications, such as distant movement of silicone, granuloma, cellulitis, monstrous nodules, and chronic ulcers. We report the case of a 65-year-old woman who presented with skin necrosis in the lower leg after using massive silicone injection 20 years ago. At the initial stage, she showed a $5 \mathrm{~cm} \times 4 \mathrm{~cm}$ sized necrotic ulcer with calcification and dirty debris of tissues. The histopathological examination of dirty debris showed acute and chronic inflammation with necrotic tissues. The ulcerative lesion was treated successfully by minimal surgical debridement, negative pressure wound therapy (NPWT) with micronized acellular dermal matrix, and conservative dressing material. In conclusion, the form of the paste acted like a scaffold to maximize the effectiveness of micronized acellular dermal matrix, thus promoting revascularization and cellular repopulation.
\end{abstract}

Keywords: Silicone fluid injection, Hard to heal wound, Chronic wound, Acellular dermal matrix, Negative pressure wound therapy

\section{Introduction}

The replacement of soft tissue defect using artificial material injection is still a challenging field for the wound specialist. There is continuous research to develop safe, long-lasting, and predictable materials today. Among the materials used for tissue augmentation, liquid silicone has a long and infamous record as an injectable artificial material for soft tissue replacement. Many complications of using liquid silicone for surface contour expansion and volume replacement of the face and body have been reported in the literature [1]. Complications are usually caused by massive injection and the use of industrial silicone and mixed impurity materials. These complications include distant movement of silicone, granuloma, cellulitis, monstrous nodules, and chronic ulcers.

In the past, massive industrial silicone was injected into humans by unauthorized personnel empirically. After silicone injection, there had been some patients who complained of late complications such as chronic ulcers. We report a case of 65 -yearold woman who complained of skin necrosis in the right lower leg after silicone injection. The patient's necrotic wound healed successfully using micronized acellular dermal matrix (ADM) (CG PASTE ${ }^{\circledR}$; CG Bio Co., Seongnam, Korea) and negative pressure wound therapy (NPWT).

\section{Case Report}

20 years ago, a 65-year-old woman with diabetes mellitus was injected with unauthor-

\author{
(4) \\ Case Report \\ Received: April 14, 2018 \\ Revised: June 28, 2018 \\ Accepted: June 29, 2018

\section{Corresponding author:} \\ Hwan Jun Choi, M.D. \\ Department of Plastic and Reconstructive \\ Surgery, College of Medicine, Soonchunhyang \\ University, 31 Suncheonhyang 6-gil, Dongnam- \\ gu, Cheonan 31151, Korea \\ Tel: +82-41-570-2195 \\ Fax: +82-41-574-6133 \\ E-mail: medi619@hanmail.net
}

This is an Open Access article distributed under the terms of the Creative Commons Attribution Non-Commercial License (http://creativecommons.org/licenses/by-nc/4.0/) which permits unrestricted non-commercial use, distribution, and reproduction in any medium, provided the original work is properly cited.

C 2018 Korean Wound Management Society 
ized industrial silicone oil in both lower legs for soft tissue expansion by non-specialist. After a few years, the injection site showed redness and swelling which subsided spontaneously and repeatedly. These symptoms repeated several times for two decades. The injection site was getting harder and looked like a chronic inflammation. Most recently, three months ago, her symptoms in right lower leg recurred and she was referred to our department. Two years ago, this patient had already been referred to our clinic for inflammation of the lateral malleolar area of the contralateral leg (left). The left leg wound was successfully treated with Pelnac ${ }^{\circledR}$ (Gunze Corp., Osaka, Japan), an artificial dermis, and a split-thickness skin graft. A few months after discharge, the patient was referred to our clinic again. On visiting our clinic this time, she showed $5 \times 4 \mathrm{~cm}$ sized ulcerated wound with skin necrosis, calcification and dirty debris of tissues (Fig. 1) on her right lower leg. After ad-

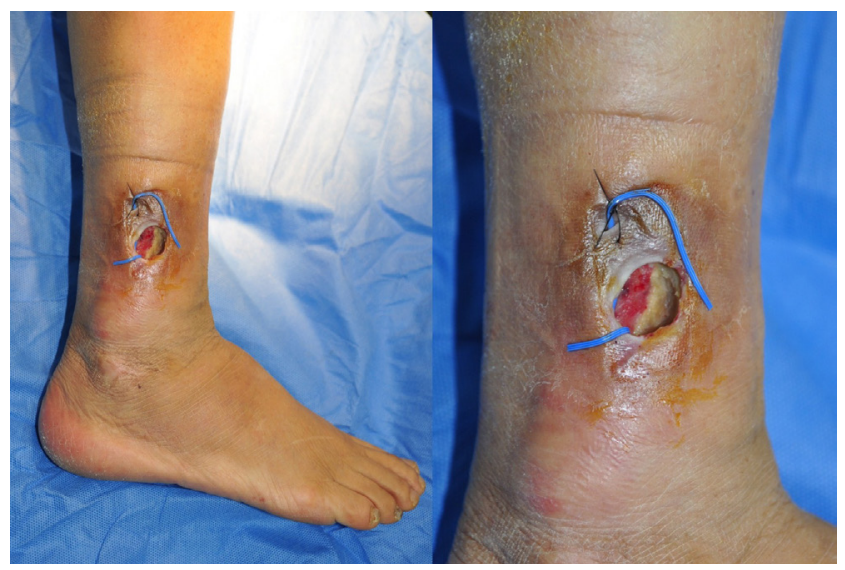

Fig. 1. This photograph shows initial skin defect and calcific dirty debris of tissues on wound base and margin.

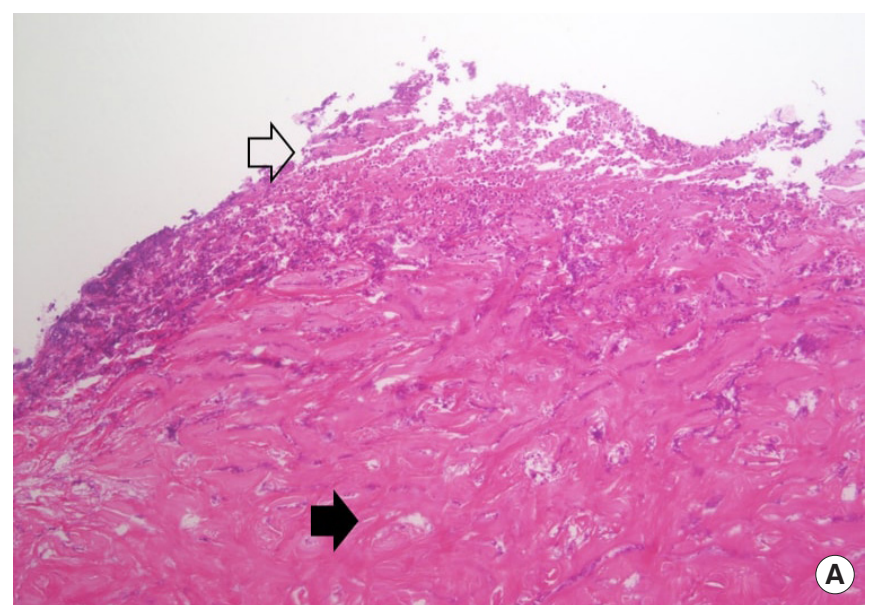

mission, we took the patient's lower extremity angio-computed tomography (CT) to evaluate vessel occlusion, for free flap coverage operation. On lower extremity angio CT, there appeared multiple calcifications on subcutaneous layer of whole thigh \& lower leg (Fig. 2). Additionally, we took tissue culture from dirty debris of tissue to check for bacterial infection. Dirty debris turned into a hard block as a result of chronic inflammation with subcutaneous fat and muscle fascia and developed a distinct boundary with the surrounding tissue. In tissue culture, there was also no evidence of bacterial growth. The histopathological examination of the biopsy specimens collected from dirty debris reported acute and chronic inflammation with surrounding calcification. The specimen consisted of fibrotic tissue covered with necrotic debris and neutrophilic aggregation and variable sized silicon globules were dispersed (Fig. 3). In this patient, we planned conservative treat-
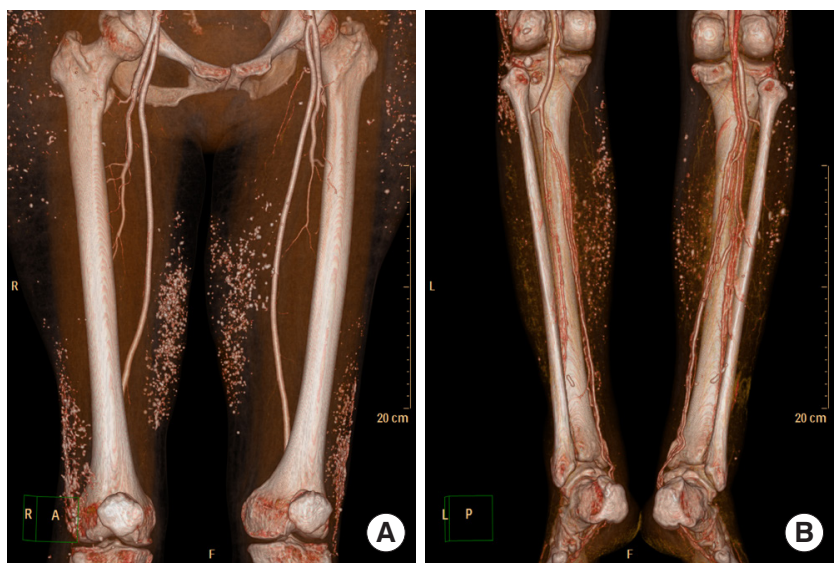

Fig. 2. The lower extremity angio CT shows multiple calcification on both thigh \& lower leg $(A, B)$.

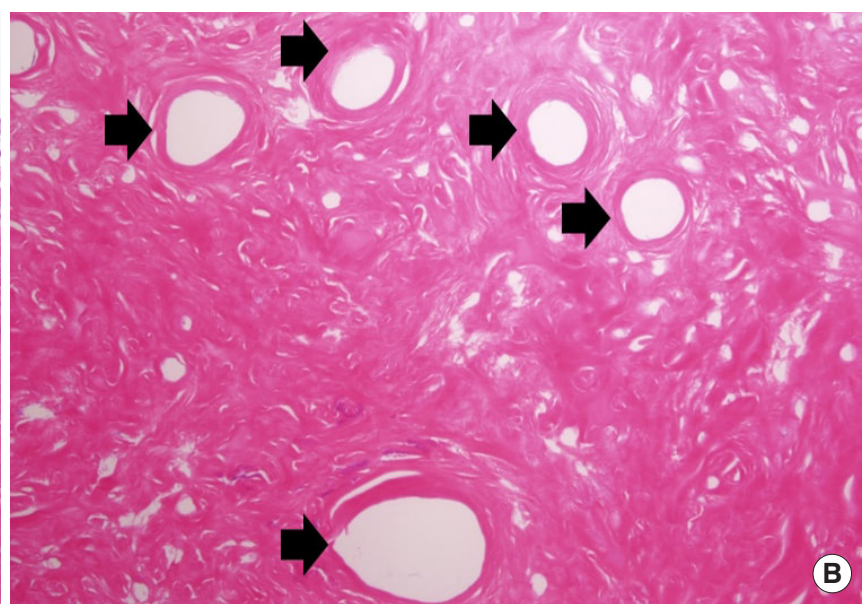

Fig. 3. (A) The specimen consisted of fibrotic tissue (black arrow) covered with necrotic debris and neutrophilic aggregation (white arrow) $(H \& E, \times 40)$. (B) The variable sized silicone globules (black arrow) were dispersed within fibrotic tissue (H\&E, $\times 200)$. 
ment rather than surgical treatment, such as flap coverage and skin graft. Firstly, there was hard calcification of surrounding tissue around whole lower leg due to chronic inflammation and there was no tissue elasticity suitable for local flap coverage. Even though angio CT revealed no evidence of vascular occlusion, no appropriate recipient vessels for free flap coverage could be found due to chronic inflammation. As industrial silicone was found in nearly the entire lower leg level, curettage was performed only on infected areas, and the silicone could not be totally removed. Other adjacent areas were prone to develop inflammation. And she strongly rejected operation and preferred conservative treatment. Secondly, we had a history of successful treatment of the contralateral leg (left) of the patient using an artificial dermis such as Pelnac ${ }^{\circledR}$. Hence, we tried to apply a similar treatment using artificial dermis for this wound. Thus, several consecutive surgical debridements and NPWT (Cura VAC ${ }^{\circledR}$; CG Bio Co., Seongnam, Korea) were
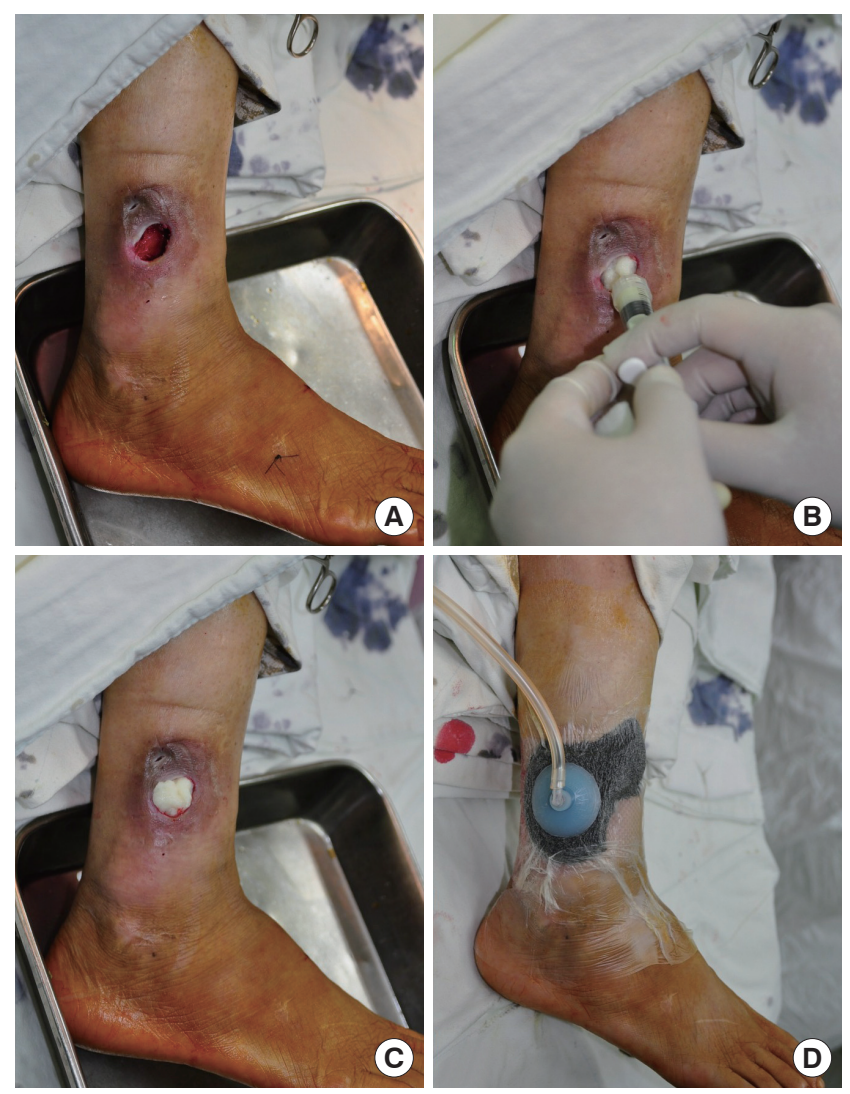

Fig. 4. (A) After serial debridement, healthy granulation tissue was visible in the bottom. (B, C) After irrigation and preparation of the wound, the skin and soft tissue defect site was filled in with diced acellular dermal matrix (CG PASTE ${ }^{\circledR}$ ). (D) On the wound filled with CG PASTE ${ }^{\circledR}$, negative pressure wound therapy (NPWT, Cura VAC) was applied. undertaken, until healthy tissue was seen inside the wound. We used micronized ADM (CG PASTE ${ }^{\circledR}$ ) and directly applied it to the wound (Fig. 4). The micronized ADM was manufactured by adding gelatin to sliced CGDERM ${ }^{\circledR}$ and NPWT device was installed above micronized ADM. After replacing the NPWT three times a week for two weeks, growth of healthy granulation tissue and perfusion of gross wound margin was confirmed in the vertical and horizontal planes.

Subsequently, we planned to use polyurethane foam material for wound dressing and not to perform split-thickness skin graft. As a result of maintaining the dressing treatment, the wound healed after three months. One-year after follow-up, she had no complications (Fig. 5). The patient was subjectively satisfied with the final result, and the wound did not cause any functional disability, such as gait disturbance.

\section{Discussion}

Soft tissue expansion using the injection of silicone has been used for the past 30 years [2]. Improvement of body contour

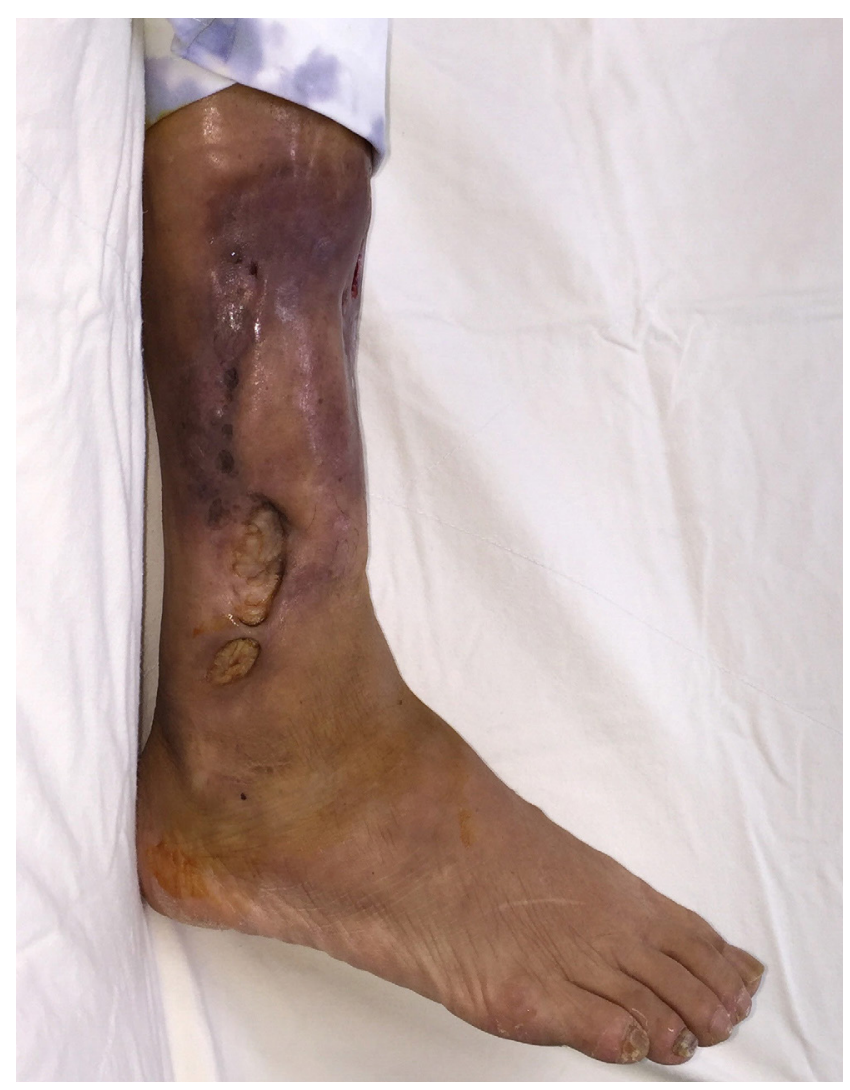

Fig. 5. After 1 year follow up, skin and soft tissue defect on the right lower leg was healed and well epithelized. No complications occurred. 
using liquid silicone was first introduced in Germany, Switzerland, and Japan in the 1940s, and the first decade seemed to make use of silicone actively. However, evidences of not only safety but also long-term effects in human body are still insufficient. Furthermore, the patients complain of side effects such as formation of tender nodules and ulcerations due to the inflammatory reaction between the silicone and human immune system.

Rapaport et al. [3] mentioned that these side effects were due to the following: contaminated materials, physician's poor technique, injection of too much volume, and too superficial injection. Thus, although silicone is chemically inert as from known journals, this is not a biologically inert material. For this reason, it may cause a chronic inflammation. Rowe et al. [4] first confirmed foreign body granuloma caused by the injection of liquid silicone in 1948. Around the granuloma they observed the fibrous capsule with increased fibroblasts. The inflammatory response around the wound was very serious and all affected tissues were excised widely. In our case, the immune responses caused by silicone migration appeared in the patient's lower leg with multiple calcified and periodic inflammations. As a result, necrotized skin tissue defect and silicone induced granuloma appeared. There was a report that the silicone granuloma may occur as fast as 6 years after silicone injection as long as 11 years [3]. In our case, we observed chronic wound that occurred 20 years after silicone injection. At first, we did biopsy and prove there are numerous cyst with silicone glonules. The patient in our case was injected with too much volume of impure silicone with physician's poor technique. This point appears to be related to the occurrence of complications.

Until now there is controversy about the treatment of silicone induced ulcer. Steroid medication and liposuction have been tested, but results are not usually successful. Total excision of the affected area is also definite treatment. However, there is a problem that the wound becomes too larger and deeper. Hence, the excised wound usually requires flap reconstruction [5]. Lee et al. [2] reported a similar patient who complained of silicone induced ulcerated wound and treated the wound successfully with surgical debridement and Penlac ${ }^{\circledR}$. It was accepted and published in other journals. In our case, silicone granuloma like a hard block was excised focally with minimal surgical debridement. We used NPWT device applied with micronized ADM and conservative foam material to induce wound healing in the defected area of the excised tissue, and the wound healing occurred successfully. We also use Intravenous antibiotics for few days. Antibiotics were not used for long periods of time because there were no signs of a general infection during several hospitalization and the CRP levels were low.

The dermal extracellular matrix (ECM) has proteins that assist structural and signaling functions critical for skin wound healing. But the ECM of chronic wound is easily degraded by wound's inflammatory and proteolytic environment. Since ECM is often destroyed in chronic wounds, various materials have been devised to promote healing of damaged or deformed ECM [6]. ADM is one such type among them.

The use of ADM offers several advantages compared with other bioengineered skin grafts. First, the skin graft is an immunologically inert, acellular, replacement scaffold derived from human cadaver tissue and is comprised of collagen and extracellular protein matrices. Furthermore, it provides a favorable microenvironment for bio-ingrowth by promoting nutritional diffusion and cellular proliferation for complete wound healing. These properties result in the rapid revascularization and cellular repopulation of the matrix scaffold [7].

Gelatin is a protein biopolymer derived from native collagen, which is a structural protein that occupies the most part of the human skin, tendon, cartilage, and bone. Gelatin has several advantages such as biological origin, non-immunogenicity, biodegradability, biocompatibility, and relatively low cost [8]. Recently, there is a report on gelatin-related phagocytosis and high hemostatic effect. Finally, gelatin is more comfortable to use than collagen [9]. For this reason, the gelatin has been widely used in the pharmaceutical and medical fields. Ulubayram, et al. [10] reported that gelatin sponges contain epithelial growth factor and have a significant effect on reducing the size of wound and improvement of inflammatory signs of tissue damage in experimentally created skin lesions in a rabbit.

To the best of our knowledge, the majority of studies previously published on the outcomes of ADM have involved freeze-drying using processed materials such as Alloderm ${ }^{\circledR}$ (Life Cell Co., Branchburg, NJ, USA). Histologically micronized AlloDerm ${ }^{\circledR}$ helps in wound healing with fibroblast ingrowth and collagen deposition in a stable environment. Because of these characteristics, micronized AlloDerm ${ }^{\circledR}$ has better effect on skin wounds compared to injectable bovine collagen and even AlloDerm ${ }^{\circledR}$ sheets [11]. CG PASTE ${ }^{\circledR}$ can have side benefits of both acellular dermal matrix and gelatin.

A controlled long-term study is needed to answer whether a high volume of liquid silicone injection causes permanent for- 
eign body reaction, which results in skin and subcutaneous fat tissue scarring due to chronic inflammation. Consequently, venous drainage is impaired and elevates the possibility of venous ulcer and foreign body reaction. Thus, a close follow-up is needed. The incurable wound treatment with micronized ADM may be considered as another alternative wound dressing method.

\section{Acknowledgment}

This study was supported by the Soonchunhyang University Research Fund.

\section{References}

1. Rae V, Pardo RJ, Blackwelder PL, et al. Leg ulcers following subcutaneous injection of a liquid silicone preparation. Arch Dermatol 1989;125:670-3.

2. Lee JH, Choi HJ. Rare complication of silicone fluid injection presenting as multiple calcification and skin defect in both legs a case report. Int J Low Extrem Wounds 2015:14:95-7.

3. Rapaport MJ, Vinnik C, Zarem H. Injectable silicone: cause of facial nodules, cellulitis, ulceration, and migration. Aesthetic Plast Surg 1996;20:267-76.

4. Rowe, V.K., Spencer, H., Bass,S., Toxicology studies on certain commercial silicones and hydrolysable silane intermedi- ates, J. Industr. Hyg. and Toxicology 1948;30:332.

5. Bigatà X, Ribera M, Bielsa I, et al. Adverse granulomatous reaction after cosmetic dermal silicone injection. Dermatol Surg 2001;27:198-200.

6. Kirsner RS, Bohn G, Driver VR, et al. Human acellular dermal wound matrix: evidence and experience. Int Wound J 2015;12:646-54.

7. Reyzelman A, Crews RT, Moore JC, et al. Clinical effectiveness of an acellular dermal regenerative tissue matrix compared to standard wound management in healing diabetic foot ulcers: a prospective, randomized, multicenter study. Int Wound J 2009;6:196-208.

8. Zhang Y, Venugopal J, Huang ZM, et al. Crosslinking of the electrospun gelatin nanofibers. Polymer 2006;47:2911-7.

9. Choi YS, Hong SR, Lee YM, et al. Study on gelatin-containing artificial skin: I. Preparation and characteristics of novel gelatin-alginate sponge. Biomaterials 1999;20:409-17.

10. Ulubayram K, Cakar AN, Korkusuz P, et al. EGF containing gelatin-based wound dressings. Biomaterials 2001;22:134556.

11. Sclafani AP, Romo T, Jacono AA, et al. Evaluation of acellular dermal graft in sheet (AlloDerm) and injectable (micronized AlloDerm) forms for soft tissue augmentation: clinical observations and histological analysis. Arch Plast Surg 2000; 2:130-6. 